\title{
Long-term prognosis of clinically early lgA nephropathy is not always favorable
}

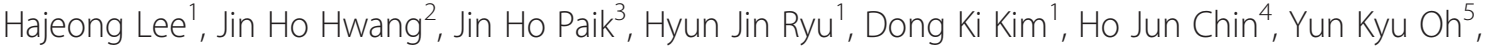 \\ Kwon Wook Joo ${ }^{1}$, Chun Soo Lim ${ }^{5}$ Yon Su Kim and Jung Pyo Lee
}

\begin{abstract}
Background: The long-term prognosis of clinically early IgA nephropathy (IgAN) patients remains to be clarified. We investigated the long-term outcomes of IgAN patients with an apparently benign presentation and evaluated prognostic factors for renal survival.

Methods: We included patients with biopsy-proven IgAN who had estimated glomerular filtration rates (eGFR) $\geq 60 \mathrm{~mL} / \mathrm{min} / 1.73 \mathrm{~m}^{2}$, normal blood pressure, and proteinuria $<0.5 \mathrm{~g} /$ day at the time of biopsy. The primary outcome was progression to end-stage renal disease (ESRD). The secondary outcome was a 50\% increase in serum creatinine level or an increase in proteinuria to $>1 \mathrm{~g} /$ day.

Results: The analysis included 153 patients who met the inclusion criteria. At diagnosis, their median systolic blood pressure was $120(110-130) \mathrm{mmHg}$, eGFR was $85.9(74.9-100.1) \mathrm{mL} / \mathrm{min} / 1.73 \mathrm{~m}^{2}$, and proteinuria was $0.25(0.13-0.38) \mathrm{g} /$ day. Of these, 4 patients died and 6 reached ESRD. The 30-year renal survival rate was $85.5 \%$. Three patients had increased serum creatinine levels and 11 developed proteinuria. Remission was observed in 35 (22.9\%) patients. A moderate or severe degree of interstitial fibrosis (adjusted odd ratio [OR] 5.93, 95\% confidence interval $[\mathrm{Cl}] 1.44-24.45, P=0.014$ ) and hypoalbuminemia (adjusted $\mathrm{OR} 6.18,95 \% \mathrm{Cl} 1.20-31.79, P=0.029$ ) were independent predictors of the secondary outcome.

Conclusions: This study showed that the prognosis of early IgAN was not always favorable, even resulting in progression to ESRD in some cases. Hypoalbuminemia and interstitial fibrosis should also be considered important prognostic factors in clinically early IgAN patients.
\end{abstract}

Keywords: IgA nephropathy, Interstitial fibrosis, Progression of renal failure

\section{Background}

IgA nephropathy (IgAN) is a common glomerular disease and an important cause of kidney failure. This disease accounts for more than half of all forms of primary glomerulonephritis in Korea [1]. Although dominant mesangial IgA deposits represent the diagnostic hallmark of IgAN, its clinical features are highly variable, ranging from simple hematuria with or without proteinuria to a rapidly progressive loss of renal function. Therefore, the renal survival and risk factors of longterm IgAN patients have been studied extensively over the last 30 years [2]. Previous studies indicate that the

\footnotetext{
* Correspondence: nephrolee@gmail.com

${ }^{5}$ Department of Internal Medicine, Seoul National University Boramae

Medical Center, Seoul, Korea

Full list of author information is available at the end of the article
}

likelihood of dialysis or death can be estimated using three clinical risk factors: urinary protein excretion of more than $1 \mathrm{~g} /$ day, high blood pressure exceeding $140 / 90 \mathrm{mmHg}$, and a decreased estimated glomerular filtration rate (eGFR) of less than $60 \mathrm{~mL} / \mathrm{min} / 1.73 \mathrm{~m}^{2}$ [3]. Among patients with all three risk factors, about $70-80 \%$ reached end-stage renal disease (ESRD) and 45\% died within 30 years $[4,5]$. These clinical prognostic factors also independently predict a poor clinical course. However, information on the long-term outcomes of IgAN patients with a minor presentation is scarce.

Only a few studies have focused on long-term patient and renal outcomes in IgAN patients who had a renal biopsy performed for microscopic hematuria, normal renal function, and minimal proteinuria. Moreover, the results of previous studies are controversial. A recently 
published European cohort study found that IgAN patients who presented with minor urinary abnormalities and normal renal function did not progress to ESRD, and that more than one-third of patients achieved clinical remission [6]. Research performed in Hong Kong, however, found that $33 \%$ of patients with minimal proteinuria and preserved renal function developed more than $1 \mathrm{~g}$ /day of proteinuria [7,8]. In a cohort of Chinese IgAN patients with isolated microscopic hematuria who were followed for up to 12 years, a decrease in renal function was observed in $24 \%$ [9]. These studies suggest that IgAN patients require long-term follow-up due to the potential for progressive disease.

Our previously, reported findings on the mortality of IgAN patients, which were obtained from our longterm follow-up data [4], did not significantly differ from European data [10]. The present study focused on longterm outcomes and prognostic factors for renal survival in clinically early IgAN patients. We hypothesized that even patients presenting with benign manifestations would progress during long-term follow-up.

\section{Methods}

\section{Ethics statement}

Ethical approval was obtained from the Institutional Review Board of Seoul National University Hospital (H-1010-055-336), and this study was conducted in accordance with the principles of the Declaration of Helsinki. As the study was retrospective in design and did not involve any interventions, the Institutional Review Board waived informed consent for this study.

\section{Study subjects}

From 1979 to 2012, a total of 1,613 adult patients were diagnosed with IgAN based on immunofluorescence microscopy showing mesangial IgA deposition as the predominant or co-dominant immunoglobulin. We included IgAN patients with preserved renal function (eGFR $\geq 60 \mathrm{~mL} / \mathrm{min} / 1.73 \mathrm{~m}^{2}$ ) and minimal proteinuria (urinary protein-to-creatinine ratio $<0.5 \mathrm{~g} / \mathrm{g}$ creatinine or 24-h urinary protein $<0.5 \mathrm{~g} /$ day). We excluded patients with fewer than 5 glomeruli per biopsy section and those in whom IgAN was the result of secondary causes, as indicated by clinical or laboratory evidence of systemic lupus erythematosus, Henoch-Schonlein nephritis, or liver cirrhosis. Patients who were followed for less than 12 months were also excluded. Ultimately, a total of 153 remaining patients were analyzed (Figure 1).

\section{Clinical parameters}

Clinical information was collected from a review of computerized medical records. Demographic factors, including age, sex, body mass index, and blood pressure at the time of renal biopsy, were obtained. Blood and urine

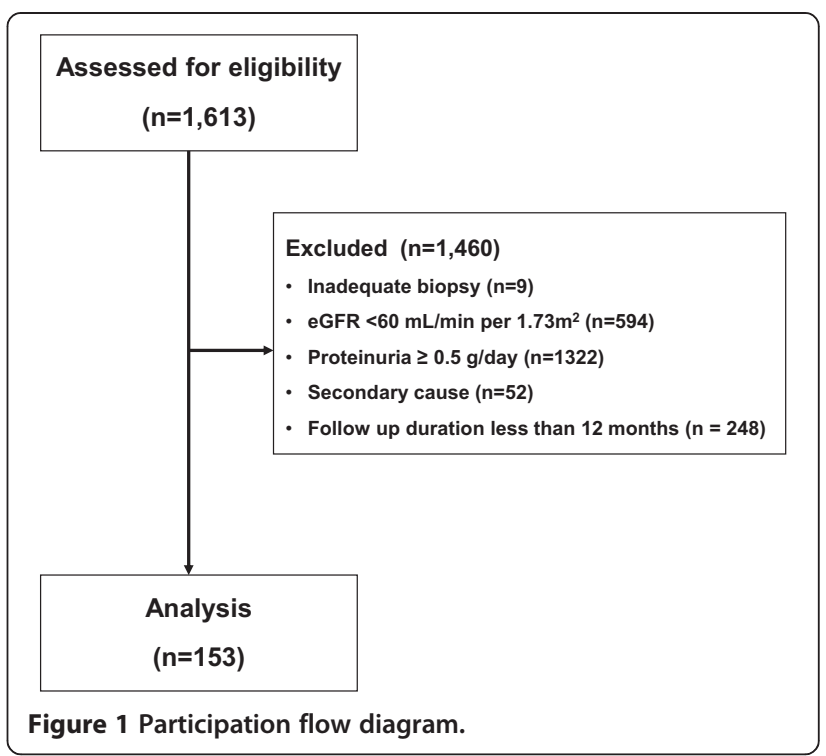

chemistry parameters were extensively reviewed from the time of renal biopsy to the time of last follow-up. Until 2010, serum creatinine levels were measured by Jaffe kinetic alkaline picrate method using a Hitachi 7600 analyzer (Toshiba, 200FR, Tokyo, Japan). Serum creatinine levels were re-calibrated to an isotope-dilution mass spectrometry assay (Roche diagnostic). The correction equation is as follows: recalibrated serum creatinine $=1.0734 \mathrm{x}$ measured serum creatinine ${ }^{(-0.2418)}$. The eGFR was calculated using the Modification of Diet in Renal Disease equation [11,12]. Proteinuria was assessed using either a random urine protein to creatinine ratio (normal range, $<0.2 \mathrm{~g} / \mathrm{g}$ creatinine) or 24-h urinary protein measurement. Information on treatments prescribed during the follow-up period was collected, including exposure to immunosuppressive agents and to renin-angiotensin system (RAS) blockade by angiotensin-converting enzyme inhibitors or angiotensin receptor blockers.

\section{Pathological parameters}

All native renal biopsies were processed according to light microscopy, immunofluorescence, and electron microscopy standard techniques. Histopathological changes were evaluated by, two pathologists who reviewed the renal biopsy slides. All IgAN biopsies were staged according to the WHO grading system [13]. The proportions of global sclerosis, segmental sclerosis, and crescent formation in the glomerular area were calculated using results obtained on light microscopy. Mesangial proliferation was also graded as none or minimal, mild, moderate, and severe. In the tubulointerstitial area, the degrees of tubular atrophy (TA), interstitial fibrosis (IF), and interstitial inflammation were graded semi-quantitatively as follows: none or minimal, mild, moderate, and severe. 


\section{Outcome measurement}

The primary outcome was ESRD progression or patient death. The start of the follow-up period was taken as the date of renal biopsy. ESRD was defined as progression to eGFR $<15 \mathrm{~mL} / \mathrm{min} / 1.73 \mathrm{~m}^{2}$, initiation of permanent dialysis, or kidney transplantation. Data on mortality were obtained from the Korean National Statistical Office, and those on ESRD were collected from the Korean ESRD registry $[14,15]$. We combined all these data according to the unique identification number issued to all Koreans. The secondary outcome was renal progression, defined as a $50 \%$ serum creatinine increase or the development of proteinuria greater than $1 \mathrm{~g} /$ day. We also evaluated patient clinical remission, defined as the disappearance of microscopic hematuria confirmed on more than 3 occasions, proteinuria persistently lower than $0.2 \mathrm{~g} /$ day, and normal renal function.

\section{Statistical analysis}

The data are presented as frequencies and percentages for categorical variables. Continuous variables are shown as medians and interquartile ranges (IQR). Comparisons between the outcome group and other groups were performed using the $\chi^{2}$ test for dichotomous variables and the Mann-Whitney test for asymmetric continuous variables. Cumulative renal survival was determined by the Kaplan-Meier method. Non-renal deaths were excluded from the renal survival rate analysis. The renal survival time for each patient was computed from the time of renal biopsy to the last follow-up. Associations between baseline variables and outcomes were tested using logistic regression. Potential confounding variables identified a priori included hypoalbuminemia (serum albumin level, <3.5 g/dL), RAS blockade, and pathological changes such as segmental sclerosis, interstitial fibrosis, and tubular atrophy. Variables that showed a significant association $(p<0.10)$ in the univariate analysis or were of considerable theoretical relevance were entered into the multivariate stepwise logistic regression models. Analyses were performed using the SPSS software package (version 20.0, Chicago, IL, USA). All tests were two-tailed, with $P$-values $<0.05$ considered statistically significant.

\section{Results}

\section{Baseline characteristics}

Considering the exclusion criteria described above, a total of $153(9.5 \%)$ patients were included in the final analyses. The median follow-up duration was 95 (38207) months. Patient demographic information is summarized in Table 1. The age at the time of biopsy was 26 (20-36) years, and $43.8 \%$ of patients were male. The initial eGFR was $85.9(74.9-100.1) \mathrm{mL} / \mathrm{min} / 1.73 \mathrm{~m}^{2}$ and systolic blood pressure was $120(110-130) \mathrm{mmHg}$. The median value of proteinuria was $0.25(0.13-0.38) \mathrm{g} /$ day.
Approximately $43 \%$ of the patients were prescribed RAS blockers during the follow-up period. No patient was treated with immunosuppressive agents including corticosteroids.

In the histopathological review, more than half of the patients showed minimal or mild pathological change while $47(30.7 \%)$ patients had advanced pathological change, with WHO grade higher than III. Glomerular crescent formation was found in $9.2 \%$ of the patients. Moreover, more than $12 \%$ of patients had a moderate to severe degree of mesangial proliferation, TA or IF.

\section{Primary outcomes}

During the observation period, 6 patients developed ESRD and 4 patients died. The 30-year renal survival rate was $85.5 \%$ (Figure 2). The details of patients who reached to ESRD are described in Table 2. Although they had preserved renal function and minimal proteinuria at the time of biopsy, half of the patients had an advanced pathological grade relative to their clinical manifestation. Two patients had segmental sclerosis, and one had moderate degree of TA/interstitial inflammation. None of the patients who reached ESRD showed crescent formation or vascular structural change. Time to ESRD ranged from 5 to 19 years. Four patients died. One patient died subsequent to ESRD progression. However, the remaining 3 patients died with functioning kidneys. The patient in whom renal failure progressed to ESRD 153 months following kidney biopsy, died 142 months after peritoneal dialysis was initiated. The cause of death was colonic pseudo-obstruction and intestinal perforation. Causes of death in the patients who had died with functioning kidneys were traffic accident in one case, ovarian cancer in one case, and unknown in the other cases.

\section{Secondary outcomes}

Secondary outcomes could be analyzed in 118 of the 153 patients, for whom renal function and urinalysis data were available. Three patients showed a greater than $50 \%$ increase of serum creatinine levels compared with those at baseline. Eleven patients developed proteinuria with protein levels of at least $1.0 \mathrm{~g}$ /day during the follow-up period. The baseline clinical characteristics of progressive patients were similar to those of stable patients (Table 3). However, patients who met the secondary outcome had a higher frequency of segmental sclerosis $35.7 \%$ vs $8.7 \%, P=0.012$ ), IF of more than a moderate degree (35.7\% vs $7.8 \%, P=0.009)$, and RAS blockade use ( $84.6 \%$ vs $45.4 \%, P=0.009)$.

\section{Predictors of outcome}

Table 4 shows the results of predictor analyses according to secondary and composite outcomes. For the primary outcome, we failed to identify any clinical or 
Table 1 Baseline clinical characteristics of early IgAN patients

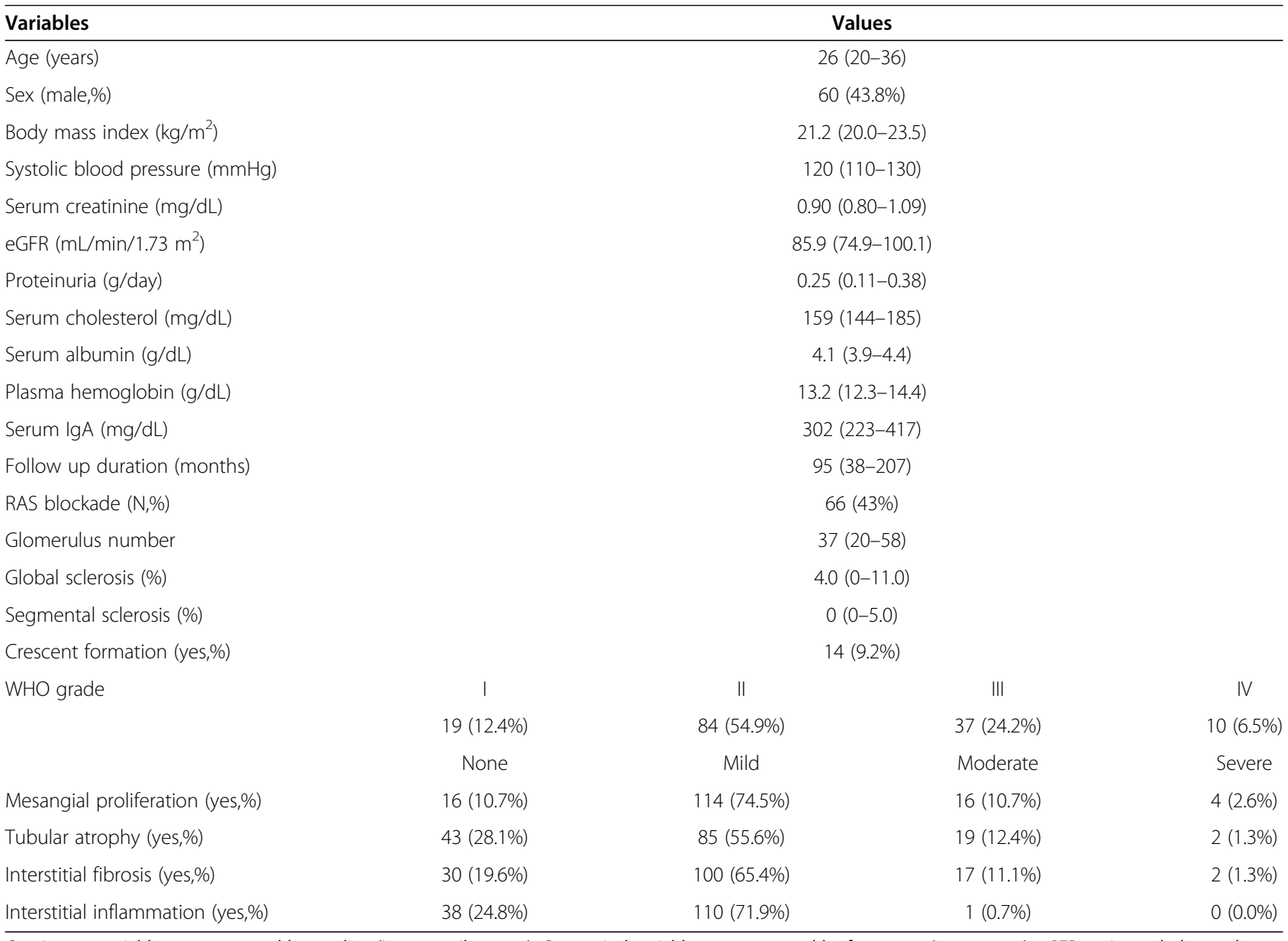

Continuous variables are presented by median (interquartile range). Categorical variables are presented by frequency (percentage). eGFR, estimated glomerular filtration rate; RAS, renin-angiotensin system.

pathological risk factors. In the univariate analysis, hypoalbuminemia $<3.5 \mathrm{~g} / \mathrm{dL}$, global and segmental sclerosis, IF, TA, and RAS blockader use were identified as potential predictors of the secondary outcomes. After the multivariate analysis, only hypoalbuminemia (odds ratio [OR] $11.89,95 \% \mathrm{CI} 2.10-67.23, P=0.005)$ and IF (OR 5.93, 95\% CI 1.44-24.45, $P=0.014$ ) remained as independent risk factors. Regression analyses of a composite of the primary and secondary outcomes showed similar results to analyses of the secondary outcome. Both hypoalbuminemia (OR 6.18, 95\% CI 1.20-31.79, $P=0.029$ ) and IF (OR 3.82, 95\% CI 1.11-13.11, $P=0.033$ ) remained independent determinants of the composite outcome.

\section{Clinical remission}

During follow-up, 36 (31.0\%) patients showed loss of proteinuria or microscopic hematuria along with stable blood pressure and renal function, in other words, clinical remission. Patients who achieved clinical remission had low systolic blood pressures (median [IQR], 114
[110-124] mmHg vs 120 [110-120] mmHg), less TA (11.4\% vs $12.5 \%)$ and less IF (8.6\% vs $11.3 \%)$. However, these differences were all statistically non-significant. Only the amount of proteinuria $(0.28$ [0.13-0.41] vs 0.21 [0.09-0.33], $P=0.021)$ and prescription of RAS blockade $(34.3 \%$ vs $56.3 \%, \mathrm{P}=0.042)$ differed significantly according to clinical remission. Our regression analysis did not find any predictors of clinical remission (data not shown).

\section{Discussion}

The clinical course of IgAN is highly variable. However, it is essential to determine whether a patient is at high risk for renal insufficiency in order to establish an individualized management plan. Forecasting the prognosis of benign IgAN on the basis of the currently known prognostic factors or modeling systems is challenging. Experience gathered over a sufficient observation period is essential to address these patients' renal survival. Based on our long follow-up period, we demonstrated that 


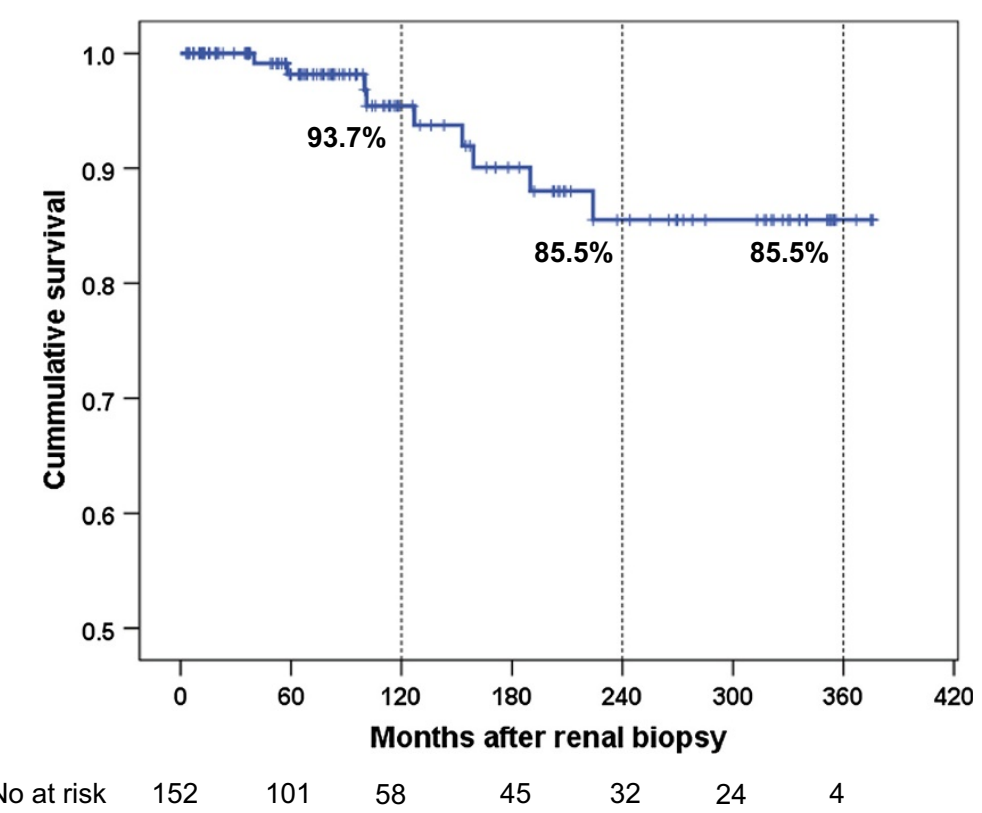

Figure 2 Cumulative renal and patient survival after kidney biopsy in early IgAN patients. The primary endpoint is free of death or end stage renal disease. The number of patients remaining at 60,120, 180, 240, 300 and 360 months of follow-up are shown at the bottom.

even clinically early IgAN patients can show a progressive disease trajectory in Korea. More than moderate degree of IF and hypoalbuminemia were independent predictors of renal progression. This study is uniquely placed to clarify the prognosis of clinically early IgAN.
Our results are similar to those of previous Chinese and Japanese studies $[8,9,16,17]$, and are contrary to those of a recent European study [6] (Table 5). In particular, our results evoke awareness of the fact that, even with a clearly benign initial presentation, IgAN patients can

Table 2 Clinical manifestation of patients who reached to end-stage renal disease

\begin{tabular}{|c|c|c|c|c|c|c|}
\hline & Patient A & Patient B & Patient C & Patient D & Patient E & Patient $\mathrm{F}$ \\
\hline Body mass index $\left(\mathrm{kg} / \mathrm{m}^{2}\right)$ & 24.6 & Unknown & 18.3 & 21.6 & 23.2 & 21.4 \\
\hline Blood pressure (mmHg) & $110 / 10$ & $130 / 90$ & $100 / 70$ & $110 / 70$ & $110 / 70$ & $100 / 60$ \\
\hline Blood urea nitrogen (mg/dL) [10-26] & 9 & 17 & 12 & 12 & 12 & NA \\
\hline Serum creatinine (mg/dL) [0.70-1.40] & 1.00 & 1.20 & 0.90 & 1.20 & 0.70 & 1.30 \\
\hline Creatinine clearance (mL/min) & 78.5 & NA & 84 & 87 & NA & 66 \\
\hline $\mathrm{eGFR}\left(\mathrm{mL} / \mathrm{min} / 1.73 \mathrm{~m}^{2}\right)$ & 72.1 & 74.1 & 77.1 & 82.7 & 152.4 & 74.6 \\
\hline Serum albumin (g/dL) [3.3-5.2] & 4.6 & 4.9 & 3.5 & 4.4 & 4.0 & NA \\
\hline Proteinuria (g/day) & 0.21 & 0.22 & 0.35 & 0.34 & 0.18 & 0.20 \\
\hline WHO grade & III & $\|$ & $\|$ & III & III & NA \\
\hline Global sclerosis (N/Glom No) & $0 / 19$ & $1 / 15$ & $0 / 8$ & $2 / 32$ & $1 / 35$ & $0 / 5$ \\
\hline Segmental sclerosis (N/Glom No) & $0 / 19$ & $0 / 15$ & $0 / 8$ & $2 / 32$ & $2 / 35$ & $0 / 5$ \\
\hline Crescent formation (N/Glom No) & $0 / 19$ & $0 / 15$ & $0 / 8$ & $0 / 32$ & $0 / 35$ & $0 / 5$ \\
\hline Mesangial proliferation & None & Mild & None & Mild & Mild & Moderate \\
\hline Tubular atrophy & Mild & None & None & Moderate & Mild & NA \\
\hline Interstitial fibrosis & None & None & None & Mild & Mild & NA \\
\hline Interstitial inflammation & Mild & None & Mild & Moderate & None & NA \\
\hline Vascular change & None & None & None & None & None & None \\
\hline Time to ESRD (months) & 153 & 224 & 101 & 100 & 58 & 159 \\
\hline
\end{tabular}


Table 3 Comparison between patients with secondary renal outcome and those without

\begin{tabular}{|c|c|c|c|}
\hline Variables & Renal outcome (-) & Renal outcome (+) & $P$-value \\
\hline N & 104 & 14 & \\
\hline Age at the time of biopsy (years) & $26(19-37)$ & $27(25-35)$ & 0.640 \\
\hline Sex (male, \%) & $47 / 104(45.2 \%)$ & 3/14 (21.4\%) & 0.148 \\
\hline Body mass index $\left(\mathrm{kg} / \mathrm{m}^{2}\right)$ & $21.6(19.9-23.5)$ & $21.7(20.5-23.1)$ & 0.749 \\
\hline Systolic blood pressure (mmHg) & $120(110-126)$ & $115(110-130)$ & 0.990 \\
\hline Serum creatinine (mg/dL) & $0.90(0.80-1.04)$ & $0.80(0.70-0.90)$ & 0.016 \\
\hline eGFR $\left(\mathrm{ml} / \mathrm{min} / 1.73 \mathrm{~m}^{2}\right)$ & $86.4(74.9-105.6)$ & $93.0(82.5-113.2)$ & 0.142 \\
\hline Albumin (g/dL) & $4.1(3.9-4.4)$ & $3.9(3.5-4.3)$ & 0.038 \\
\hline Proteinuria (g/day) & $0.25(0.10-0.40)$ & $0.28(0.10-0.41)$ & 0.680 \\
\hline WHO grade & $2.0(2.0-2.0)$ & $2.5(2.0-3.0)$ & 0.047 \\
\hline Global sclerosis $\geq 10 \%$ & $32 / 104(30.8 \%)$ & 8/14 (57.1\%) & 0.214 \\
\hline Segmental sclerosis $\geq 10 \%$ & 9/104 (8.7\%) & $5 / 14(35.7 \%)$ & 0.012 \\
\hline Cresent & 10/104 (9.6\%) & $1 / 14(7.1 \%)$ & 1.000 \\
\hline Mesangial proliferation & 15/103 (14.6\%) & $3 / 14(21.4 \%)$ & 0.450 \\
\hline Interstitial fibrosis & $8 / 103(7.8 \%)$ & $5 / 14(35.7 \%)$ & 0.009 \\
\hline Interstitial inflammation & $1 / 103(1.0 \%)$ & $0 / 14(0.0 \%)$ & 1.000 \\
\hline Tubular atrophy & 11/103 (10.7\%) & $5 / 14(35.7 \%)$ & 0.024 \\
\hline RAS blockade (N, \%) & 48/103 (45.4\%) & 12/14 (84.6\%) & 0.009 \\
\hline
\end{tabular}

Table 4 Univariate and multivariate logistic regression analysis for development of outcome

\begin{tabular}{|c|c|c|c|}
\hline Variables & Wald & OR $(95 \% \mathrm{Cl})$ & $P$-value \\
\hline \multicolumn{4}{|l|}{ Secondary outcome } \\
\hline \multicolumn{4}{|l|}{ Univariate } \\
\hline Hypoalbuminemia & 9.68 & $13.33(2.61-68.19)$ & 0.002 \\
\hline Global sclerosis & 3.58 & $3.00(0.96-9.36)$ & 0.058 \\
\hline Segmental sclerosis & 7.23 & $5.86(1.62-21.29)$ & 0.007 \\
\hline Interstitial fibrosis & 7.97 & $6.60(1.78-24.45)$ & 0.005 \\
\hline Tubular atrophy & 5.72 & 4.65 (1.32-16.37) & 0.017 \\
\hline Use of RAS blockade & 5.97 & $6.88(1.47-32.27)$ & 0.015 \\
\hline \multicolumn{4}{|l|}{ Multivariate analysis } \\
\hline Hypoalbuminemia & 7.85 & $11.89(2.10-67.23)$ & 0.005 \\
\hline Interstitial fibrosis & 6.06 & $5.93(1.44-24.45)$ & 0.014 \\
\hline \multicolumn{4}{|l|}{ Composite outcome } \\
\hline \multicolumn{4}{|l|}{ Univariate } \\
\hline Hypoalbuminemia & 5.99 & $7.19(1.48-34.86)$ & 0.014 \\
\hline Segmental sclerosis & 2.89 & $2.84(0.85-9.47)$ & 0.089 \\
\hline Interstitial fibrosis & 5.85 & $4.31(1.32-14.09)$ & 0.016 \\
\hline Tubular atrophy & 3.72 & $3.03(0.98-9.47)$ & 0.054 \\
\hline \multicolumn{4}{|l|}{ Multivariate analysis } \\
\hline Hypoalbuminemia & 4.75 & $6.18(1.20-31.79)$ & 0.029 \\
\hline Interstitial fibrosis & 4.53 & $3.82(1.11-13.11)$ & 0.033 \\
\hline
\end{tabular}

Continuous variables are presented by median (interquartile range). Categorical variables are presented by frequency (percentage). eGFR, estimated glomerular filtration rate; RAS, renin-angiotensin system. have a malignant renal or patient outcome. In this cohort, one in every 25 early IgAN patients progressed to ESRD during their lifetimes. Such an observation has not been reported previously. Considering that baseline characteristics among previous studies were similar, the cause of the varying prognosis of clinically early IgAN requires investigation. One possible explanation is the difference in the definition of outcomes. In the Chinese studies, renal progression was defined only in terms of worsening and not in relation to initial renal function [8,9]. Conversely, the European study defined renal progression as an increase in serum creatinine levels of $\geq 50 \%$ from initial levels or ESRD progression. However, our study separated ESRD progression and impaired renal function, and our definition of impaired renal function was derived from the European study. Our data indicate that the prognosis of early IgAN patients is relatively worse than that of similar European IgAN patients even when a comparable outcome definition is applied.

The divergence in findings may also be explained by racial differences. Recent publications suggest that an Asian racial origin could be identified as a risk for disease progression in IgAN $[18,19]$. Despite the fact that our study and the European study enrolled early IgAN patients with similar baseline renal function and proteinuria levels and used similar outcome definitions, renal prognosis in our cohort was considerably different from that observed in the European cohort. To clarify the influence of ethnicity on these observations, a delicate 
Table 5 Comparisons of definition and outcome of early IgAN with the previous studies

\begin{tabular}{|c|c|c|c|c|c|}
\hline & Hong Kong ('00) & Hong Kong ('01) & China ('08) & Europe ('12) & Korea ('14) \\
\hline N & $45(N A)$ & $72(10 \%)$ & $177(\mathrm{NA})$ & 141 & $153(8.8 \%)$ \\
\hline \multirow[t]{3}{*}{ Definition } & $\mathrm{Cr}<1.3 \mathrm{mg} / \mathrm{dL}$ & $\mathrm{Cr}<120 \mu \mathrm{mol} / \mathrm{L}$ & $\mathrm{eGFR}>90 \mathrm{~mL} / \mathrm{min}$ & $\mathrm{eGFR}>60 \mathrm{~mL} / \mathrm{min}$ & $\mathrm{eGFR}>60 \mathrm{~mL} / \mathrm{min}$ \\
\hline & Proteinuria $<0.4 \mathrm{~g} /$ day & Proteinuria $<0.4 \mathrm{~g} /$ day & Proteinuria $<0.4 \mathrm{~g} /$ day & Proteinuria $<0.5 \mathrm{~g} /$ day & Proteinuria $<0.5 \mathrm{~g} /$ day \\
\hline & HTN (-) & HTN (-) & HTN (-) & & HTN (-) \\
\hline FU months & $123(60-180)$ & $84(14-112)$ & 111 (109-205) & $108(60-180)$ & $95(38-207)$ \\
\hline Age (years) & $29(15-57)$ & $27(15-50)$ & $38 \pm 16$ & $23(5-71)$ & $26(16-65)$ \\
\hline \multicolumn{6}{|l|}{ Outcome } \\
\hline ESRD & No & 1 patient & No & No & 6 patients \\
\hline $\mathrm{Cr} \uparrow$ & $6(13 \%)$ & $5(7 \%)$ & $43(24 \%)$ & $5(3.5 \%)$ & $3(2.1 \%)$ \\
\hline Proteinuria $\uparrow$ & $15(34 \%)$ & $24(33 \%)$ & $79(46 \%)$ & $21(14.9 \%)$ & $11(18.3 \%)$ \\
\hline HTN & $11(24 \%)$ & $19(26 \%)$ & $68(38 \%)$ & $23(16.3 \%)$ & NA \\
\hline Remission & NA & $10(14 \%)$ & $10(6 \%)$ & $53(38 \%)$ & $36(25 \%)$ \\
\hline
\end{tabular}

NA, not applicable; $\mathrm{Cr}$, creatinine; eGFR, estimated glomerular filtration rate; HTN, hypertension; ESRD, end stage renal disease.

genetic analysis with consideration of phenotype should be performed.

Lead-time bias may also explain the different outcomes in early IgAN. Because of variations in biopsy practices, the disease is detected at different times in its natural course. Even among early IgAN patients with similar initial presentations, the duration of disease could be different. Some patients visit the clinic immediately after gross hematuria or incidentally detected hematuria, while others visit several years after the initial manifestation. However, clinicians can only conduct assessments at the time of initial visit or biopsy. Therefore, a comparison of these patients using only initial data could be limited by lead-time bias.

Our data also showed that pathological changes including IF can be important for renal risk prediction in clinically early IgAN patients. More than $12 \%$ of patients showed a more than moderate degree of tubulointerstitial changes. Furthermore, IF is an independent predictor of ESRD progression in this study. Such results are consistent with some previous studies $[9,20,21]$ and inconsistent with another study [6]. Although IgAN is a glomerular disease, tubulo-interstitial injury via the mesangio-podocytic-tubular crosstalk plays an important role in mediating renal fibrosis and, ultimately resulting in, renal failure [22]. In our study, mesangial hypercellularity or interstitial inflammation was not associated with renal progression. Those are considered relatively early renal injury markers, whereas IF is regarded as relatively advanced marker in IgAN patients with minor abnormalities. Indeed, IF is one component of the Oxford classification, although it is validated mostly in patients with proteinuria of more than $1 \mathrm{~g} /$ day $[23,24]$. Our study results support the applicability of the Oxford classification even in IgAN patients with a minimal clinical presentation.
We further demonstrated that hypoalbuminemia is a significant predictor of renal outcome. These finding is consistent with those in previous IgAN studies $[4,25]$ as well as those in other CKD studies [26,27]. Lower serum albumin levels can be explained by the amount of proteinuria, nutritional status or combined inflammation. In our cohort, 7 patients showed reduced serum albumin levels. Their proteinuria amount was ranged between 0.16 and $0.45 \mathrm{~g} /$ day. Three patients had combined inflammation, and one patient suffered from tuberculosis and was subsequently malnourished. In other words, hypoalbuminemia may contribute to a poor renal outcome, independently from proteinuria.

The particular strengths and insights gained from this investigation include the long follow-up duration and large sample size, which is specifically important in IgAN research because of the insidious course of this disease. We were able to assess hard outcomes such as ESRD progression and mortality in this study. Moreover, we were able to suggest racial differences in IgAN prognosis by using baseline characteristics and outcome definitions similar to those used in the prior European study. We were also able to alarm many nephrologists and primary physicians who have managed clinically early IgAN patients with ease, especially in Korea.

However, several shortcomings remain to be resolved. First, the number of outcomes observed in our cohort was too small despite the long follow-up duration. Lack of events hampered our ability to perform robust multivariate predictive modeling. Second, this is a singlecenter, retrospective study, and therefore, we could not take into account diverse management strategies according to individual clinicians. Although hard outcome data were collected by both medical record review and from a national registry or statistics, the last clinical 
status remains unknown in some cases. Third, we could not use the Oxford classification in this study. However, we were able to measure each component of the Oxford classification semi-quantitatively, as shown in Table 1. Lastly, we could not clarify the precise reason for progression to ESRD among patients with initially benign clinical presentations. We proposed different outcome definitions, lead-time bias, and ethnicity as potential explanations. However, we could not prove these, and further sequential investigation is therefore warranted.

\section{Conclusions}

In this study, we demonstrated that clinically early IgAN does not always show a favorable outcome and may even progress to ESRD. Patients with IF and hypoalbuminemia should be more aggressively monitored, especially in Korea.

\section{Abbreviations}

IgAN: IgA nephropathy; eGFR: Estimated glomerular filtration rate; ESRD: End-stage renal disease; RAS: Renin-angiotensin system;

TA: Tubular atrophy; IF: Interstitial fibrosis; IQR: Interquartile ranges; OR: Odds ratio; $\mathrm{Cl}$ : Confidence interval.

\section{Competing interest}

The authors declare that they have no competing interests.

\section{Authors' contributions}

All authors contributed extensively to the work presented in this paper at all stage. HL, HJC and JPL conceived the design of this research and wrote the manuscript. JPL supervised this project. KWJ and YKO assembled input data. $\mathrm{JHB}$ and JHH performed statistical analyses. HJR and CSL interpreted the data analyses. DKK and YSK gave conceptual advice and commented on the manuscript. All authors read and approved the final manuscript.

\section{Acknowledgements}

This study was supported by a grant from the Korea Healthcare Technology R\&D Project, Ministry for Health and Welfare, Republic of Korea (HI10C2020).

\section{Author details}

${ }^{1}$ Department of Internal Medicine, Seoul National University Hospital, Seoul, Korea. ${ }^{2}$ Department of Internal Medicine, Chung-Ang University Hospital, Seoul, Korea. ${ }^{3}$ Department of Pathology, Seoul National University Bundang Hospital, Seongnam, Korea. ${ }^{4}$ Department of Internal Medicine, Seoul National University Bundang Hospital, Seongnam, Korea. ${ }^{5}$ Department of Internal Medicine, Seoul National University Boramae Medical Center, Seoul, Korea.

\section{Received: 7 January 2014 Accepted: 13 June 2014}

Published: 19 June 2014

\section{References}

1. Lee H, Kim DK, Oh KH, Joo KW, Kim YS, Chae DW, Kim S, Chin HJ: Mortality and renal outcome of primary glomerulonephritis in Korea: observation in 1,943 biopsied cases. Am J Nephrol 2013, 37(1):74-83.

2. Barbour SJ, Reich HN: Risk stratification of patients with IgA nephropathy. Am J Kidney Dis 2012, 59(6):865-873.

3. Wyatt RJ, Julian BA: IgA nephropathy. N Engl J Med 2013, 368(25):2402-2414.

4. Lee H, Kim DK, Oh KH, Joo KW, Kim YS, Chae DW, Kim S, Chin HJ: Mortality of IgA nephropathy patients: a single center experience over 30 years. PLoS One 2012, 7(12):e51225.

5. Kiryluk K, Novak J, Gharavi AG: Pathogenesis of immunoglobulin A nephropathy: recent insight from genetic studies. Annu Rev Med 2013, 64:339-356.
6. Gutiérrez E, Zamora I, Ballarín JA, Arce Y, Jiménez S, Quereda C, Olea T, Martínez-Ara J, Segarra A, Bernis C, García A, Goicoechea M, García de Vinuesa S, Rojas-Rivera J, Praga M: Long-term outcomes of $\lg A$ nephropathy presenting with minimal or no proteinuria. J Am Soc Nephrol 2012, 23(10):1753-1760.

7. Li PK, Ho KK, Szeto CC, Yu L, Lai FM: Prognostic indicators of IgA nephropathy in the Chinese-clinical and pathological perspectives. Nephrol Dial Transplant 2002, 17(1):64-69.

8. Szeto CC, Lai FM, To KF, Wong TY, Chow KM, Choi PC, Lui SF, Li PK: The natural history of immunoglobulin a nephropathy among patients with hematuria and minimal proteinuria. Am J Med 2001, 110(6):434-437.

9. Shen P, He L, Huang D: Clinical course and prognostic factors of clinical early IgA nephropathy. Neth J Med 2002, 66(6):242-247.

10. Knoop T, Vikse BE, Svarstad E, Leh S, Reisaeter AV, Bjorneklett R: Mortality in patients with IgA nephropathy. Am J Kidney Dis 2013, 62(5):833-890.

11. Levey AS, Coresh J, Greene T, Stevens LA, Zhang YL, Hendriksen S, Kusek JW, Van Lente F: Using standardized serum creatinine values in the modification of diet in renal disease study equation for estimating glomerular filtration rate. Ann Intern Med 2006, 145(4):247-254

12. Lee CS, Cha RH, Lim YH, Kim H, Song KH, Gu N, Yu KS, Lim CS, Han JS, Kim S, Kim YS: Ethnic coefficients for glomerular filtration rate estimation by the Modification of Diet in Renal Disease study equations in the Korean population. J Korean Med Sci 2010, 25(11):1616-1625.

13. Sinniah R: IgA Mesangial Nephropathy: Berger's Disease. Am J Nephrol 1985, 5(2):73-83.

14. Jin DC: Current status of dialysis therapy in Korea. Korean J Intern Med 2011, 26(2):123-131.

15. Kim SY, Jin DC, Bang BK: Current status of dialytic therapy in Korea. Nephrology 2003, 8(Suppl):S2-S9.

16. Lai FM, Szeto CC, Choi PC, Li PK, Chan AW, Tang NL, Lui SF, Wang AY, To KF: Characterization of early IgA Nephropathy. Am J Kidney Dis 2000, 36 (4):703-708

17. Koyama A, Igarashi M, Kobayashi M: Natural history and risk factors for immunoglobulin A nephropathy in Japan. Research Group on Progressive Renal Diseases. Am J Kidney Dis 2000, 29(4):526-532.

18. Kiryluk K, Li Y, Sanna-Cherchi S, Rohanizadegan M, Suzuki H, Eitner F, Snyder HJ, Choi M, Hou P, Scolari F, Izzi C, Gigante M, Gesualdo L, Savoldi S, Amoroso A, Cusi D, Zamboli P, Julian BA, Novak J, Wyatt RJ, Mucha K, Perola M, Kristiansson K, Viktorin A, Magnusson PK, Thorleifsson G, Thorsteinsdottir U, Stefansson K, Boland A, Metzger M, et al: Geographic differences in genetic susceptibility to IgA nephropathy: GWAS replication study and geospatial risk analysis. PLOS Genet 2012, 8(6):e1002765

19. Barbour SJ, Cattran DC, Kim SJ, Levin A, Wald R, Hladunewich MA, Reich HN: Individuals of Pacific Asian origin with IgA nephropathy have an increased risk of progression to end-stage renal disease. Kidney Int 2013 84(5):1017-1024.

20. Remuzzi G, Bertani T: Pathophysiology of progressive nephropathies. N Engl J Med 1998, 339(20):1448-1456.

21. Daniel L, Saingra Y, Giorgi R, Bouvier C, Pellissier JF, Berland Y: Tubular lesions determine prognosis of IgA nephropathy. Am J Kidney Dis 2000, 35(1):13-20

22. Lai KN: Pathogenesis of IgA nephropathy. Nat Rev Nephrol 2012, 8(5):275-283

23. Cattran DC, Coppo R, Cook HT, Feehally J, Roberts IS, Troyanov S, Alpers CE, Amore A, Barratt J, Berthoux F, Bonsib S, Bruijn JA, D'Agati V, D'Amico G, Emancipator S, Emma F, Ferrario F, Fervenza FC, Florquin S, Fogo A Geddes CC, Groene HJ, Haas M, Herzenberg AM, Hill PA, Hogg RJ, Hsu SI Jennette JC, Joh K, Julian BA, et al: The Oxford classification of IgA nephropathy: rationale, clinicopathological correlations, and classification. Kidney Int 2009, 76(5):534-545.

24. Kang SH, Choi SR, Park HS, Lee JY, Sun IO, Hwang HS, Chung BH, Park CW, Yang CW, Kim YS, Choi YJ, Choi BS: The Oxford classification as a predictor of prognosis in patients with IgA nephropathy. Nephrol Dial Transplant 2012, 27(1):252-258.

25. Xie J, Kiryluk K, Wang W, Wang Z, Guo S, Shen P, Ren H, Pan X, Chen X, Zhang W, Li X, Shi H, Li Y, Gharavi AG, Chen N: Predicting progression of IgA nephropathy: new clinical progression risk score. PLOS One 2012, 7(6):e38904 
26. Tangri N, Stevens LA, Griffith J, Tighiouart H, Djurdjev O, Naimark D, Levin A, Levey AS: A predictive model for progression of chronic kidney disease to kidney failure. JAMA 2011, 305(15):1553-1559.

27. Keane WF, Brenner BM, De Zeeuw D, Grunfeld JP, McGill J, Mitch WE, Ribeiro AB, Shahinfar S, Simpson RL, Snapinn SM, Toto R: The risk of developing end-stage renal disease in patients with type 2 diabetes and nephropathy: the RENAAL study. Kidney Int 2003, 63(4):1499-1507.

doi:10.1186/1471-2369-15-94

Cite this article as: Lee et al.: Long-term prognosis of clinically early IgA nephropathy is not always favorable. BMC Nephrology 2014 15:94.

\section{Submit your next manuscript to BioMed Central and take full advantage of:}

- Convenient online submission

- Thorough peer review

- No space constraints or color figure charges

- Immediate publication on acceptance

- Inclusion in PubMed, CAS, Scopus and Google Scholar

- Research which is freely available for redistribution 